trials (RCTs) assessing the efficacy of psychological therapies for adults with IBS. Trials included in the analysis reported a dichotomous assessment of symptom status after completion of therapy ( $\geq 4$ weeks), and data were pooled using a random effects model. We examined 6 and 12-month outcomes, where reported. Efficacy was reported as a pooled relative risk (RR) of remaining symptomatic, with a 95\% confidence interval (CI) to summarise efficacy of each comparison tested. Treatments were ranked by therapy according to Pscore.

Results We identified 41 eligible RCTs, containing 4072 participants. At the first point of follow-up, after completion of therapy, contingency management was ranked first, but 95\% CIs were wide (RR of remaining symptomatic $=0.39 ; 95 \%$ CI 0.19 to 0.84 , P-score 0.89 ), and this was based on only one small RCT (figure 1). The psychological interventions with the largest numbers of trials, and patients recruited, included self-administered or minimal contact $\mathrm{CBT}(\mathrm{RR}=$ $0.61 ; 95 \%$ CI 0.45 to 0.83 , P-score 0.66), face-to-face CBT $(\mathrm{RR}=0.62 ; 95 \% \mathrm{CI} 0.48$ to $0.80, \mathrm{P}$ score 0.65$)$, and gutdirected hypnotherapy $(\mathrm{RR}=0.67 ; 95 \% \mathrm{CI} 0.49$ to 0.91 , Pscore 0.57). CBT-based interventions and gut-directed hypnotherapy were the most efficacious long-term. Risk of bias of individual trials was high, meaning that the efficacy of all psychological therapies studied is likely to have been overestimated.

Conclusions Several psychological therapies are efficacious for IBS, although none were superior to another. CBT-based interventions and gut-directed hypnotherapy had the largest evidence base. Future RCTs should examine the effect of psychological therapy earlier in the disease course, before patients are refractory to medical management.

\section{O62 PSYLLIUM REDUCES COLONIC HYDROGEN PRODUCTION FOLLOWING INGESTION OF INULIN IN IRRITABLE BOWEL SYNDROME}

\begin{abstract}
1,2 David Gunn* 1,2Zainab Abbas, 1,2 Giles Major, 1,3 Caroline Hoad, 1,3Penny Gowland, 1,2 Luca Marciani, ${ }^{4}$ Hannah Harris, ${ }^{4}$ Fred Warren, ${ }^{5}$ Samantha Gill, ${ }^{5}$ Megan Rossi, ${ }^{6}$ Jose Remes-Troche, ${ }^{5}$ Kevin Whelan, ${ }^{1,2}$ Robin Spiller. ${ }^{1}$ NIHR Nottingham Biomedical Research Centre, Nottingham University Hospitals NHS Trust and the University of Nottingham, Nottingham, UK; ${ }^{2}$ Nottingham Digestive Diseases Centre, School of Medicine, University of Nottingham, Nottingham, UK; ${ }^{3}$ Sir Peter Mansfield Imaging Centre, University of Nottingham, Nottingham, UK; ${ }^{4}$ Quadram Institute Biosciences, Food, Innovation and Health, Norwich Research Park, Norwich, UK; ${ }^{5}$ King's College London, Department of Nutritional Sciences, London, UK; ${ }^{6}$ Digestive Physiology and Motility Lab, Medical Biological Research Institute, University of Veracruz, Veracruz, Mexico
\end{abstract}

\subsection{6/gutjnl-2020-bsgcampus.62}

Introduction Patients with irritable bowel syndrome (IBS) often develop symptoms of gas and flatulence after intake of the prebiotic inulin, leading to dietary avoidance that may have deleterious effects on gut microbiota. Our aim was to determine if co-administering inulin with psyllium, a viscous virtually non-fermentable fibre, known to improve symptoms in IBS, would increase viscosity in the ascending colon and slow fermentation, therefore reducing gas production.

Methods A randomised, four-period, four-treatment, placebocontrolled, crossover trial of 19 patients with IBS (meeting Rome IV criteria, 10 diarrhoea- and 9 constipation-predominant). Patients followed a standardised low-fibre diet on the day preceding each visit then fasted overnight prior to MRI investigations. Interventions were ingested as $500 \mathrm{ml}$ drinks containing either inulin $20 \mathrm{~g}$, psyllium $20 \mathrm{~g}$, inulin $20 \mathrm{~g}+$ psyllium $20 \mathrm{~g}$ or placebo (dextrose) $20 \mathrm{~g}$. A $446 \mathrm{kcal}$ meal was consumed after 3 hours. Breath hydrogen and GI symptoms were recorded every 30 minutes and MRI scanning was performed hourly for 6 hours.

Results Breath hydrogen rose significantly from 120 minutes after inulin; the addition of psyllium strikingly reduced this rise (51 [95\% CI 33-69] ppm versus 18 [95\% CI 6/30] ppm at 360 minutes, $p=0.0004)$. Psyllium alone or dextrose produced no significant rise. At the end of the study, patients reported significantly less flatulence with inulin + psyllium than inulin alone $(p=0.008)$. The rise in small bowel water content was highest after psyllium ingestion, peaking at 3 hours. Co-administration of inulin with psyllium significantly reduced SBWC AUC (mean difference -15.8(95\% CI 1.530.1) 1.min, $p=0.028$ ). Colonic volumes rose steadily through the study day, peaking at 6 hours. Inulin + psyllium had the fastest rate of rise from fasting, which was significantly greater than psyllium $(0.89$ [95\%CI 0.68 to 1.1$] \mathrm{ml} / \mathrm{min}$ versus 0.39 [95\% CI 0.22 to 0.55$] \mathrm{ml} / \mathrm{min}, p=0.0004$ ) but not inulin.

Conclusions Psyllium significantly slows the fermentation of inulin, reducing the production of hydrogen and the symptoms of flatulence. This was likely due to its high viscosity reducing the access of the microbiota to inulin. Whether coadministration with psyllium increases the tolerability of prebiotics in IBS warrants a large-scale randomised controlled trial.

\section{GASTROINTESTINAL SYMPTOM-SPECIFIC ANXIETY AND SYMPTOM SEVERITY IN IRRITABLE BOWEL SYNDROME: NEW INSIGHTS FROM FACTOR ANALYSIS}

${ }^{1,2}$ Christopher Black*, ${ }^{3}$ Yan Yiannakou, ${ }^{2}$ Lesley A Houghton, ${ }^{4}$ Farag Shuweihdi, ${ }^{4}$ Robert West, ${ }^{4}$ Elspeth Guthrie, ${ }^{1,2}$ Alexander C Ford. 'Leeds Gastroenterology Institute, St. James's University Hospital, Leeds, UK; ${ }^{2}$ Leeds Institute of Medical Research at St. James's, University of Leeds, Leeds, UK; ${ }^{3}$ County Durham and Darlington NHS Foundation Trust, Durham, UK; ${ }^{4}$ Leeds Institute of Health Sciences, University of Leeds, Leeds, UK

\subsection{6/gutjnl-2020-bsgcampus.63}

Introduction Gastrointestinal symptom-specific anxiety and somatisation have both been associated with higher symptom severity in patients with irritable bowel syndrome (IBS). However, the relationship between these two factors and IBS symptom severity has not been explored fully. In addition, the performance of the instrument that measures gastrointestinal symptom-specific anxiety, the visceral sensitivity index (VSI), has not been examined in a UK population. We conducted a cross-sectional survey to examine these issues.

Methods We measured levels of gastrointestinal symptom-specific anxiety, using the VSI, somatisation via the patient health questionnaire-12 (PHQ-12), as well as symptom severity in adult subjects from the UK community with Rome IV-defined IBS. We carried out exploratory factor analysis on the VSI, prior to subsequent analyses, to establish its factor structure. We carried out multiple regression analysis to determine the relationship between demographic features, different factors of the VSI, somatisation, and IBS symptom severity.

Results 811 individuals with IBS provided complete data. The mean age was 47.4 years, and $85.9 \%$ were female. Factor analysis of the VSI revealed a three-factor structure, accounting for $47 \%$ of the variance. Three VSI items that loaded onto factor one were concerned with awareness of abdominal 